Holiday Scams: The Gift that Keeps on Taking

National Survey of Adults 18+: Annotated Questionnaire

November 2021

\title{
Screening Questions
}

Market: National

Screening Criteria: U.S. Adults 18+

Mode: Telephone survey

Sample: $n=1,027$ (The sample has been weighted by age, gender, and race/ethnicity based on 2019 5-year American Community Survey statistics.)

${ }^{*}$ Percentages may not add up to $100 \%$ due to rounding

S1. Our study is interested in the opinions of certain age groups. Could you please tell me your age as of your last birthday? Are you ......? [AGE CODED]

\begin{tabular}{|c|l|}
\hline$\%$ & $\mathbf{n}=\mathbf{1 , 0 2 7}$ \\
\hline 0 & Under 18 [TERMINATE] \\
\hline 12 & $18-24$ \\
\hline 20 & $25-34$ \\
\hline 19 & $35-44$ \\
\hline 18 & $45-54$ \\
\hline 16 & $55-64$ \\
\hline 16 & $65+$ \\
\hline 0 & Refused [TERMINATE] \\
\hline
\end{tabular}

S2. To ensure it is recorded accurately, could you please state your gender?

\begin{tabular}{|c|l|}
\hline$\%$ & $\mathbf{n}=\mathbf{1 , 0 2 7}$ \\
\hline 53 & Female \\
\hline 47 & Male \\
\hline 1 & Something else \\
\hline$<1$ & Refused \\
\hline
\end{tabular}




\section{AARP}

S3. The months November through January are often referred to as the Holiday Season. These can be ethnic, patriotic, religious, or other non-religious events. Do you shop for gifts, food, decorations, etc. specifically for a holiday or other sorts of observances during these months?

\begin{tabular}{|c|l|}
\hline$\%$ & $\mathbf{n}=\mathbf{1 , 0 2 7}$ \\
\hline 100 & Yes \\
\hline 0 & No [TERMINATE] \\
\hline 0 & Not sure [TERMINATE] \\
\hline 0 & Refused [TERMINATE] \\
\hline
\end{tabular}

S4. Do you use the internet at least occasionally - this can be through a computer, tablet or iPad, phone, or TV?

\begin{tabular}{|c|l|}
\hline$\%$ & $\mathbf{n}=\mathbf{1}, \mathbf{0 2 7}$ \\
\hline 100 & Yes \\
\hline 0 & No [TERMINATE] \\
\hline 0 & Don't know [TERMINATE] \\
\hline 0 & Refused [TERMINATE] \\
\hline
\end{tabular}

S5. Do you use a smartphone like an iPhone or Samsung Galaxy to make calls, send and receive emails or texts, and browse the internet?

\begin{tabular}{|c|l|}
\hline$\%$ & $\mathbf{n}=\mathbf{1 , 0 2 7}$ \\
\hline 92 & Yes \\
\hline 8 & No \\
\hline$<1$ & Not sure \\
\hline$<1$ & Refused \\
\hline
\end{tabular}

S6. Do you own a laptop or desktop computer or a tablet computer?

\begin{tabular}{|c|l|}
\hline$\%$ & $\mathbf{n}=\mathbf{1}, \mathbf{0 2 7}$ \\
\hline 91 & Yes \\
\hline 9 & No \\
\hline$<1$ & Not sure \\
\hline$<1$ & Refused \\
\hline
\end{tabular}




\section{AARP}

\section{Main}

\section{Holiday Shopping Behavior Last Year (2020) and This Year (2021)}

Q1. Do you have plans to do any shopping, either online or in a store, on the following days of this year....? [RANDOMIZE]

\begin{tabular}{|l|c|c|c|c|}
\hline \multicolumn{1}{|c|}{$\mathbf{n = 1 , 0 2 7}$} & $\begin{array}{c}\text { Yes } \\
\mathbf{( \% )}\end{array}$ & $\begin{array}{c}\text { No } \\
(\%)\end{array}$ & $\begin{array}{c}\text { Don't } \\
\text { know } \\
(\%)\end{array}$ & $\begin{array}{c}\text { Refused } \\
\text { (\%) }\end{array}$ \\
\hline $\begin{array}{l}\text { Cyber Monday (which is the Monday after } \\
\text { Thanksgiving)? }\end{array}$ & 54 & 33 & 13 & $<1$ \\
\hline $\begin{array}{l}\text { Black Friday (which is the Friday after } \\
\text { Thanksgiving)? }\end{array}$ & 53 & 37 & 10 & $<1$ \\
\hline $\begin{array}{l}\text { Small Business Saturday (which is the Saturday } \\
\text { after Thanksgiving)? }\end{array}$ & 42 & 39 & 19 & $<1$ \\
\hline
\end{tabular}

Q2. Thinking about last holiday season (last November through January) did you shop for any holiday or event gifts or items...? [RANDOMIZE]

\begin{tabular}{|c|c|c|c|c|}
\hline$n=1,027$ & $\begin{array}{l}\text { Yes } \\
(\%)\end{array}$ & $\begin{array}{l}\text { No } \\
(\%)\end{array}$ & $\begin{array}{c}\text { Don't } \\
\text { know } \\
(\%)\end{array}$ & $\begin{array}{l}\text { Refused } \\
\text { (\%) }\end{array}$ \\
\hline $\begin{array}{l}\text { in person at a major retailer like Target, Best Buy, } \\
\text { or Kohls? }\end{array}$ & 70 & 26 & 3 & $<1$ \\
\hline $\begin{array}{l}\text { from a major online store like Amazon, Walmart or } \\
\text { eBay? }\end{array}$ & 81 & 18 & 1 & $<1$ \\
\hline $\begin{array}{l}\text { from online marketplaces like Etsy, Facebook's } \\
\text { Marketplace, or Craig's list? }\end{array}$ & 37 & 59 & 4 & $<1$ \\
\hline $\begin{array}{l}\text { in person at a specialty retailer or a local small } \\
\text { business? }\end{array}$ & 70 & 25 & 4 & $<1$ \\
\hline $\begin{array}{l}\text { online at a specialty retailer or a local small } \\
\text { business? }\end{array}$ & 60 & 35 & 5 & $<1$ \\
\hline
\end{tabular}




\section{AARP}

Q3. Compared to last holiday season (again last November through January), do you plan to purchase more, about the same, or less of your holiday or event gifts or items this holiday season....? [RANDOMIZE; REVERSE RESPONSE OPTIONS]

\begin{tabular}{|l|c|c|c|c|c|}
\hline \multicolumn{1}{|c|}{$\mathbf{n = 1 , 0 2 7}$} & $\begin{array}{c}\text { More } \\
\text { (\%) }\end{array}$ & $\begin{array}{c}\text { About } \\
\text { the } \\
\text { same } \\
(\%)\end{array}$ & $\begin{array}{c}\text { Less } \\
\text { (\%) }\end{array}$ & $\begin{array}{c}\text { Don't } \\
\text { know } \\
\text { (\%) }\end{array}$ & $\begin{array}{c}\text { Refused } \\
\text { (\%) }\end{array}$ \\
\hline $\begin{array}{l}\text { in person at a major retailer like Target, } \\
\text { Best Buy, or Kohls? }\end{array}$ & 16 & 54 & 25 & 4 & 1 \\
\hline $\begin{array}{l}\text { from a major online store like Amazon, } \\
\text { Walmart or eBay? }\end{array}$ & 21 & 54 & 21 & 4 & $<1$ \\
\hline $\begin{array}{l}\text { from online marketplaces like Etsy, } \\
\text { Facebook's Marketplace, or Craig's list? }\end{array}$ & 11 & 44 & 34 & 9 & 1 \\
\hline $\begin{array}{l}\text { in person at a specialty retailer or a } \\
\text { local small business? }\end{array}$ & 20 & 53 & 22 & 5 & $<1$ \\
\hline $\begin{array}{l}\text { online at a specialty retailer or a local } \\
\text { small business? }\end{array}$ & 16 & 55 & 22 & 6 & 1 \\
\hline
\end{tabular}

Q4. In the past 12 months, or since last October, have you purchased a product or service through clicking on an advertisement you saw on social media - like Facebook, Instagram, Twitter?

\begin{tabular}{|c|l|}
\hline$\%$ & $\mathbf{n}=\mathbf{1 , 0 2 7}$ \\
\hline 38 & Yes \\
\hline 60 & No \\
\hline 2 & Don't know \\
\hline 0 & Refused \\
\hline
\end{tabular}

Q5. Have you ever experienced fraud when seeking to buy a product through an online ad? For example, you purchased something you never received, or your payment method was compromised? (IF NECESSARY, can explain with: by 'compromised' we mean your payment method and information was revealed to an unauthorized party or person')

\begin{tabular}{|c|l|}
\hline$\%$ & $\mathbf{n = 1}, \mathbf{0 2 7}$ \\
\hline 35 & Yes \\
\hline 62 & No \\
\hline 3 & Don't know \\
\hline$<1$ & Refused \\
\hline
\end{tabular}




\section{AARP}

Q6. During this holiday season, will you purchase any of your gifts or items...? [RANDOMIZE]

\begin{tabular}{|l|c|c|c|c|}
\hline \multicolumn{1}{|c|}{$\mathbf{n = 1 , 0 2 7}$} & $\begin{array}{c}\text { Yes } \\
(\%)\end{array}$ & $\begin{array}{c}\text { No } \\
(\%)\end{array}$ & $\begin{array}{c}\text { Don't } \\
\text { know } \\
(\%)\end{array}$ & $\begin{array}{c}\text { Refused } \\
(\%)\end{array}$ \\
\hline with a debit card? & 69 & 28 & 3 & $<1$ \\
\hline with a credit card? & 67 & 27 & 5 & 1 \\
\hline with cash? & 67 & 27 & 5 & $<1$ \\
\hline with personal checks? & 13 & 83 & 4 & 1 \\
\hline Through an electronic check from your bank? & 17 & 78 & 5 & $<1$ \\
\hline in some other way (specify): & 7 & 82 & 10 & $<1$ \\
\hline
\end{tabular}

\section{P2P Use for Holiday Shopping}

Q7. Peer-to-Peer (or P2P) payment platforms allow consumers to transfer money from their bank account, credit card or debit card to another individual through the Internet or a mobile device. Please tell me if you have ever used the following types of P2P payment services:

\begin{tabular}{|l|c|c|c|c|}
\hline $\mathbf{n = 1 , 0 2 7}$ & $\begin{array}{c}\text { Yes } \\
(\%)\end{array}$ & $\begin{array}{c}\text { No } \\
(\%)\end{array}$ & $\begin{array}{c}\text { Don't } \\
\text { know } \\
(\%)\end{array}$ & $\begin{array}{c}\text { Refused } \\
(\%)\end{array}$ \\
\hline Venmo & 37 & 61 & 2 & $<1$ \\
\hline Zelle & 31 & 66 & 2 & $<1$ \\
\hline Cash App & 34 & 65 & 1 & $<1$ \\
\hline
\end{tabular}

Q8. [If Q7=Yes] And about how often have you used [that/those] P2P payment [platform/platforms] to send money to the following people...? [ROTATE AND INSERT a-c] Would you say frequently, sometimes, rarely, or never? [REVERSE RESPONSE OPTIONS EVERY OTHER RESPONDENT]?

\begin{tabular}{|l|c|c|c|c|c|c|}
\hline \multicolumn{1}{|c|}{$\mathbf{n = 6 0 4}$} & $\begin{array}{c}\text { Frequently } \\
(\%)\end{array}$ & $\begin{array}{c}\text { Sometimes } \\
(\%)\end{array}$ & $\begin{array}{c}\text { Rarely } \\
(\%)\end{array}$ & $\begin{array}{c}\text { Never } \\
(\%)\end{array}$ & $\begin{array}{c}\text { Don't } \\
\text { know } \\
(\%)\end{array}$ & $\begin{array}{c}\text { Refused } \\
\text { (\%) }\end{array}$ \\
\hline $\begin{array}{l}\text { A family member or close } \\
\text { friend? }\end{array}$ & 42 & 35 & 18 & 5 & 1 & 0 \\
\hline $\begin{array}{l}\text { An individual seller (not a } \\
\text { retailer) with whom you } \\
\text { have not previously done } \\
\text { business - a new seller? }\end{array}$ & 9 & 23 & 25 & 42 & 2 & 0 \\
\hline $\begin{array}{l}\text { Someone you know, } \\
\text { although not well? }\end{array}$ & 10 & 27 & 31 & 30 & 2 & $<1$ \\
\hline
\end{tabular}




\section{AARP}

Q9. [If Q7=Yes] How likely or unlikely are you to use a Peer-to-Peer payment service this holiday season to purchase gifts or other needs? Would you say you're very likely, somewhat likely, not too or not at all likely? [REVERSE OPTIONS EVERY OTHER RESPONDENT]

\begin{tabular}{|c|l|}
\hline$\%$ & $\mathbf{n = 6 0 4}$ \\
\hline 17 & Very likely \\
\hline 28 & Somewhat likely \\
\hline 24 & Not too likely \\
\hline 28 & Not at all likely \\
\hline 3 & Don't know \\
\hline$<1$ & Refused \\
\hline
\end{tabular}

\section{Gift Card Giving and Receiving}

Q10. Did you purchase gift cards last holiday season as presents for family and friends?

\begin{tabular}{|c|l|}
\hline$\%$ & $\mathbf{n}=\mathbf{1 , 0 2 7}$ \\
\hline 59 & Yes \\
\hline 40 & No \\
\hline 1 & Don't know \\
\hline$<1$ & Refused \\
\hline
\end{tabular}

Q11. Compared to last year's holiday season...Do you plan to buy more, about the same, fewer or no gift cards this year as presents for family and friends? [REVERSE RESPONSE

\section{OPTIONS]}

\begin{tabular}{|c|l|}
\hline$\%$ & $\mathbf{n = 1 , 0 2 7}$ \\
\hline 12 & Buy more gift cards \\
\hline 43 & Buy about the same amount of gift cards \\
\hline 11 & Buy fewer gift cards \\
\hline 29 & No gift cards at all this year [SKIP TO Q13] \\
\hline 5 & Not sure \\
\hline$<1$ & Refused [SKIP TO Q13] \\
\hline
\end{tabular}




\section{AARP}

Q12. [IF Q10=YES AND Q11=a, b, c or NS] Where will you purchase your gift cards this year's holiday season? Will you buy them....? [READ LIST; RANDOMIZE; MULTIPLE RESPONSES ALLOWED]

\begin{tabular}{|c|l|}
\hline$\%$ & $\mathbf{n = 5 6 8}$ \\
\hline 60 & From a rack at a retail, pharmacy, grocery or other store? \\
\hline 59 & At the store of a specific retailer? \\
\hline 28 & On the website of a specific retailer? \\
\hline 13 & Online through an auction, reseller, or sites like eBay or Craigslist? \\
\hline 0 & Other, specify: \\
\hline 3 & Not sure [DO NOT READ] \\
\hline 0 & Refused [DO NOT READ] \\
\hline
\end{tabular}

Q13. Have you ever given or received a gift card that ended up having no funds on it? [DO NOT READ RESPONSE OPTIONS - ACCEPT ONE RESPONSE ONLY]

\begin{tabular}{|c|l|}
\hline$\%$ & $\mathbf{n = 1 , 0 2 7}$ \\
\hline 5 & Yes, have given one \\
\hline 11 & Yes, received one \\
\hline 5 & Yes, both - given and received one with no funds on it \\
\hline 77 & No [GO TO Q15] \\
\hline 1 & Not sure [GO TO Q15] \\
\hline$<1$ & Refused [GO TO Q15] \\
\hline
\end{tabular}

Q14. [If Q13=YES] And what did you do when you discovered there were no funds on the gift card? Did you...? [RANDOMIZE; MULTIPLE RESPONSES ALLOWED]

\begin{tabular}{|c|l|}
\hline$\%$ & $\mathbf{n = 2 1 8}$ \\
\hline 47 & Call the number or visit the website noted on the back of the card? \\
\hline 32 & Ask for a manager's help at the store where you tried to redeem it? \\
\hline 36 & Contact the person who gave you the card? \\
\hline 19 & Did nothing? [VOLUNTEERED] [DO NOT READ] \\
\hline 0 & Did something else - please specify: \\
\hline 1 & Not sure [DO NOT READ] \\
\hline 1 & Refused [DO NOT READ] \\
\hline
\end{tabular}




\section{AARP}

\section{Charity Donations}

Q15. In the past 12 months, or since last October have you received a request to make a monetary donation to a charity or cause from...? [MULTIPLE RESPONSES ALLOWED]

\begin{tabular}{|c|l|}
\hline$\%$ & $\mathbf{n = 1 , 0 2 7}$ \\
\hline 41 & a phone call? \\
\hline 36 & an email? \\
\hline 39 & a letter or flyer in the postal mail? \\
\hline 24 & a text message? \\
\hline 26 & a representative or a fundraiser in person? \\
\hline 22 & a pop-up online while visiting a website? \\
\hline 31 & a social media ad or person or group you follow? \\
\hline 19 & None of the above (DO NOT READ) \\
\hline 4 & Not sure (DO NOT READ) \\
\hline$<1$ & Refused (DO NOT READ) \\
\hline
\end{tabular}

Q16. And in the past 12 months, have you donated money to a charity or cause?

\begin{tabular}{|c|l|}
\hline$\%$ & $\mathbf{n}=\mathbf{1 , 0 2 7}$ \\
\hline 60 & Yes \\
\hline 38 & No [SKIP TO QUESTION 18] \\
\hline 1 & Not sure/don't know [SKIP TO QUESTION 18] \\
\hline 1 & Refused [SKIP TO QUESTION 18] \\
\hline
\end{tabular}

Q17. Thinking back on monetary donations you made since last October, did you send money...? [RANDOMIZE; MULTIPLE RESPONSES ALLOWED]

\begin{tabular}{|c|l|}
\hline$\%$ & $\mathbf{n = 6 1 9}$ \\
\hline 8 & With a gift card? \\
\hline 30 & With a personal check? \\
\hline 54 & With a credit or debit card? \\
\hline 12 & Through an electronic check from your bank or financial institution? \\
\hline 11 & Through a Peer-to-Peer - P2P - Platform? \\
\hline 29 & With a personal check sent in the mail? \\
\hline 37 & With cash? \\
\hline 2 & Not sure (DO NOT READ) \\
\hline 1 & Refused (DO NOT READ) \\
\hline
\end{tabular}




\section{AARP}

Q18. Now thinking about all the financial contributions you've ever made to a charity or cause, would you say you tend to make monetary donations [INSERT AND REVERSE SCALE EVERY OTHER RESPONDENT] more, about the same, or less during the holiday season [USE PROPER TRANISTION: more than/same as/less than] any other time of the year?

\begin{tabular}{|c|l|}
\hline$\%$ & $\mathbf{n}=\mathbf{1 , 0 2 7}$ \\
\hline 19 & More \\
\hline 58 & About the same \\
\hline 17 & Less \\
\hline 6 & Not sure \\
\hline 1 & Refused \\
\hline
\end{tabular}

Q19. In general, would you say you [INSERT AND REVERSE SCALE EVERY OTHER RESPONDENT] always, sometimes, rarely or never research an organization or cause before making a monetary donation?

\begin{tabular}{|c|l|}
\hline$\%$ & $\mathbf{n = 1 , 0 2 7}$ \\
\hline 44 & Always \\
\hline 27 & Sometimes \\
\hline 12 & Rarely \\
\hline 14 & Never \\
\hline 3 & Not sure \\
\hline 1 & Refused \\
\hline
\end{tabular}

Q20. Where or to whom would you turn if you wanted to check to see if a charity or professional fundraiser was registered in your state or how much of a donation is used for programs and services? [DO NOT READ - USE LIST BELOW TO CODE OPEN-ENDED RESPONSES] [ALLOW ONLY FIRST/ONE RESPONSE]

\begin{tabular}{|c|l|}
\hline$\%$ & $\mathbf{n}=\mathbf{1 , 0 2 7}$ \\
\hline 2 & Attorney General's office \\
\hline 3 & Secretary of State \\
\hline 3 & Police \\
\hline 2 & Federal Trade Commission \\
\hline 14 & Better Business Bureau \\
\hline 2 & Consumer affairs group \\
\hline 2 & The Mayor's office \\
\hline 11 & Family or friends / word of mouth \\
\hline 13 & Charitynavigator.org / charitywatch.org / give.org \\
\hline 29 & Other (specify): \\
\hline 20 & Not sure \\
\hline 1 & Refused \\
\hline
\end{tabular}




\section{AARP}

Q21. In the past 12 months, have you received a request for a monetary donation to a charity or cause that you felt was likely a fake or fraudulent solicitation?

\begin{tabular}{|c|l|}
\hline$\%$ & $\mathbf{n = 1 , 0 2 7}$ \\
\hline 38 & Yes \\
\hline 54 & No \\
\hline 8 & Not sure \\
\hline$<1$ & Refused \\
\hline
\end{tabular}

\section{Packages - Porch Pirate}

Q22. This holiday season, do you plan to send any gift packages to friends or family members?

\begin{tabular}{|c|l|}
\hline$\%$ & $\mathbf{n}=\mathbf{1 , 0 2 7}$ \\
\hline 53 & Yes \\
\hline 39 & No \\
\hline 9 & Not sure \\
\hline$<1$ & Refused \\
\hline
\end{tabular}

Q23. Have you ever had a package stolen from outside your door?

\begin{tabular}{|c|l|}
\hline$\%$ & $\mathbf{n}=\mathbf{1 , 0 2 7}$ \\
\hline 25 & Yes \\
\hline 72 & No \\
\hline 3 & Not sure \\
\hline 0 & Refused \\
\hline
\end{tabular}

Q24. Have you ever received a notification from someone saying they are from USPS, FedEx, or UPS and they need to get in touch with you about a shipment issue, and it turned out to be fraudulent? [IF NECESSARY/ASKED WHAT IS MEANT BY FRAUDULENT, READ: For example, you clicked a link from a text or email you thought was legitimate, but it took you to a fake site and you picked up a software virus or your credit card was compromised.]

\begin{tabular}{|c|l|}
\hline$\%$ & $\mathbf{n}=\mathbf{1 , 0 2 7}$ \\
\hline 34 & Yes \\
\hline 62 & No \\
\hline 4 & Not sure \\
\hline$<1$ & Refused \\
\hline
\end{tabular}




\section{AARP}

Job Scam - Seasonal

Q25. Are you considering working in a temporary 'seasonal' job over this holiday season?

\begin{tabular}{|c|l|}
\hline$\%$ & $\mathbf{n}=\mathbf{1 , 0 2 7}$ \\
\hline 14 & Yes \\
\hline 79 & No \\
\hline 7 & Not sure \\
\hline$<1$ & Refused \\
\hline
\end{tabular}

Q26. Have you ever experienced fraud when applying for a seasonal holiday job...for example, paid upfront for what turned out to be a fake opportunity, or experienced identity theft or fraud after sharing sensitive personal information?

\begin{tabular}{|c|l|}
\hline$\%$ & $\mathbf{n = 1 , 0 2 7}$ \\
\hline 8 & Yes \\
\hline 89 & No \\
\hline 3 & Not sure \\
\hline$<1$ & Refused \\
\hline
\end{tabular}

\section{Consumer Scams and Fraud Knowledge Quiz}

After I read the following statement, tell me if you think it's true or false, or if you don't know or aren't sure.... let's begin. [RANDOMIZE Q27 - Q34]

Q27. Ads for merchandise that you see on social media, such as Facebook and Instagram, or other places online are trustworthy.

\begin{tabular}{|c|l|}
\hline$\%$ & $\mathbf{n = 1 , 0 2 7}$ \\
\hline 24 & True \\
\hline 53 & False \\
\hline 23 & Not sure \\
\hline 1 & Refused \\
\hline
\end{tabular}

Q28. When searching online for customer support, the first customer service phone number that appears on your search results will connect you with a legitimate person from that company.

\begin{tabular}{|c|l|}
\hline$\%$ & $\mathbf{n = 1 , 0 2 7}$ \\
\hline 30 & True \\
\hline 47 & False \\
\hline 23 & Not sure \\
\hline 1 & Refused \\
\hline
\end{tabular}




\section{AARP}

Q29. Regularly updating software on your devices provides protection against fraud.

\begin{tabular}{|c|l|}
\hline $\mathbf{n}=\mathbf{1 , 0 2 7}$ \\
\hline 60 & True \\
\hline 21 & False \\
\hline 18 & Not sure \\
\hline 1 & Refused \\
\hline
\end{tabular}

Q30. Peer-to-peer payment apps like Cash App, Zelle, or Venmo have the same consumer protections as your credit card.

\begin{tabular}{|l|l|}
\hline$\%$ & $\mathbf{n}=\mathbf{1 , 0 2 7}$ \\
\hline 25 & True \\
\hline 40 & False \\
\hline 35 & Not sure \\
\hline$<1$ & Refused \\
\hline
\end{tabular}

Q31. Peer-to-peer payment apps like Cash App, Zelle and Venmo are only intended to be used to transfer money between people you personally know, like family, friends, the babysitter, or the lawn service.

\begin{tabular}{|l|l|}
\hline$\%$ & $\mathbf{n = 1 , 0 2 7}$ \\
\hline 49 & True \\
\hline 25 & False \\
\hline 26 & Not sure \\
\hline$<1$ & Refused \\
\hline
\end{tabular}

Q32. Online retailers like Amazon and eBay will request your login information to provide customer support.

\begin{tabular}{|c|l|}
\hline $\mathbf{n}=\mathbf{1 , 0 2 7}$ \\
\hline 49 & True \\
\hline 31 & False \\
\hline 20 & Not sure \\
\hline 1 & Refused \\
\hline
\end{tabular}

Q33. Credit cards and debit cards offer the same protections when shopping online.

\begin{tabular}{|c|l|}
\hline$\%$ & $\mathbf{n}=\mathbf{1 , 0 2 7}$ \\
\hline 45 & True \\
\hline 37 & False \\
\hline 18 & Not sure \\
\hline 1 & Refused \\
\hline
\end{tabular}


Q34. Ordering a free trial offer from an online retailer (with a small shipping charge) is a good way of trying out a product before you buy it.

\begin{tabular}{|c|l|}
\hline$\%$ & $\mathbf{n = 1 , 0 2 7}$ \\
\hline 39 & True \\
\hline 43 & False \\
\hline 17 & Not sure \\
\hline 1 & Refused \\
\hline
\end{tabular}

\section{Demographics}

The following questions are for classification purposes only and will be kept entirely confidential.

D2. What is your current marital status? [READ LIST]

\begin{tabular}{|c|l|}
\hline$\%$ & $\mathbf{n = 1 , 0 2 7}$ \\
\hline 48 & Married \\
\hline 12 & Not married, living with partner \\
\hline 2 & Separated \\
\hline 8 & Divorced \\
\hline 4 & Widowed \\
\hline 26 & Never married \\
\hline$<1$ & Not sure [DO NOT READ] \\
\hline 1 & Refused [DO NOT READ] \\
\hline
\end{tabular}

D3. Do you consider yourself to be LGBTQ? [If needed, add: LGBTQ stands for lesbian, gay, bisexual, transgender, queer or questioning.]

\begin{tabular}{|c|l|}
\hline$\%$ & $\mathbf{n}=\mathbf{1 , 0 2 7}$ \\
\hline 9 & Yes \\
\hline 89 & No \\
\hline 1 & Not sure \\
\hline 1 & Refused \\
\hline
\end{tabular}


D4. What is the highest level of education that you completed? [READ LIST IF NEEDED]

\begin{tabular}{|c|l|}
\hline$\%$ & $\mathbf{n = 1 , 0 2 7}$ \\
\hline 4 & 0 -12th grade (no diploma) \\
\hline 28 & High school graduate (or equivalent) \\
\hline 5 & Post-high school education (no degree) \\
\hline 27 & Some college or 2-year college degree \\
\hline 20 & 4-year college degree \\
\hline 15 & Post-graduate study or a graduate degree \\
\hline$<1$ & Not sure [DO NOT READ] \\
\hline$<1$ & Refused [DO NOT READ] \\
\hline
\end{tabular}

D5. Which of the following best describes your current employment status? Are you...? [READ LIST]

\begin{tabular}{|c|l|}
\hline$\%$ & $\mathbf{n}=\mathbf{1 , 0 2 7}$ \\
\hline 10 & Self-employed full-time \\
\hline 5 & Self-employed part-time \\
\hline 40 & Employed full-time \\
\hline 7 & Employed part-time \\
\hline 18 & Retired and not working at all \\
\hline 10 & Unemployed and looking for work \\
\hline 9 & Or are you not in the labor force for other reasons \\
\hline 1 & Not sure [DO NOT READ] \\
\hline$<1$ & Refused [DO NOT READ] \\
\hline
\end{tabular}

D6. Are you of Hispanic, Spanish, or Latino origin or descent?

\begin{tabular}{|c|l|}
\hline$\%$ & $\mathbf{n = 1 , 0 2 7}$ \\
\hline 14 & Yes \\
\hline 85 & No \\
\hline$<1$ & Not sure \\
\hline 1 & Refused \\
\hline
\end{tabular}




\section{AARP}

D7. What is your race? Are you...? [READ LIST; ACCEPT MULTIPLE RESPONSES]

\begin{tabular}{|c|l|}
\hline$\%$ & $\mathbf{n = 1 , 0 2 7}$ \\
\hline 77 & White or Caucasian \\
\hline 12 & Black or African American \\
\hline 2 & American Indian or Alaska Native \\
\hline 5 & Asian \\
\hline 1 & Native Hawaiian or other Pacific Islander \\
\hline 4 & Other \\
\hline 1 & Not sure [DO NOT READ] \\
\hline 1 & Refused [DO NOT READ] \\
\hline
\end{tabular}

D8. We realize income is a private matter and so rather than ask you anything specific about your income, l'd first like to ask you if your household income was above or less than $\$ 30,000$ before taxes in 2020 and then l'll ask you to please stop me when I get to the category that includes your household's income. [INCOME - CODED]

\begin{tabular}{|c|l|}
\hline$\%$ & $\mathbf{n}=\mathbf{1 , 0 2 7}$ \\
\hline 4 & Less than $\$ 10,000$ \\
\hline 7 & $\$ 10,000$ to less than $\$ 20,000$ \\
\hline 11 & $\$ 20,000$ to less than $\$ 30,000$ \\
\hline 11 & $\$ 30,000$ to less than $\$ 40,000$ \\
\hline 12 & $\$ 40,000$ to less than $\$ 50,000$ \\
\hline 14 & $\$ 50,000$ to less than $\$ 75,000$ \\
\hline 13 & $\$ 75,000$ to less than $\$ 100,000$ \\
\hline 11 & $\$ 100,000$ to less than $\$ 150,000$ \\
\hline 7 & $\$ 150,000$ or more \\
\hline 3 & Not sure [DO NOT READ] \\
\hline 7 & Refused [DO NOT READ] \\
\hline
\end{tabular}

D9. What is your 5-digit ZIP Code? (Write in your ZIP CODE):

ENDING: Those are all the questions I have for you. Thank you very much for helping us with this important study. Have a great day/evening. 